\title{
METRIZABLE KÖTHE SPACES ${ }^{1}$
}

\section{R. R. WELLAND}

1. Let $E$ be a locally compact Hausdorff space, $\mu$ a Radon measure, [3], on $E$, and $\Omega(E, \mu)$ the space of equivalence classes of locally integrable functions on $E$ with respect to the measure $\mu$. Here, two functions $f$ and $g$ are equivalent if $f-g=0$ except on a set $S$ which meets every compact set in a set of measure zero; the notation $f=\lim f_{n}$ applies to equivalence classes. For a subset $\Gamma$ of $\Omega$, let

$$
\Lambda(\Gamma)=\left\{f \in \Omega:\left|\int f g d \mu\right|<\infty \text { for all } g \in \Gamma\right\} .
$$

The sets $\Lambda=\Lambda(\Gamma)$ and $\Lambda^{*}=\Lambda(\Lambda)$ are vector lattices, [1], and are called associated Köthe spaces. Initially Köthe and Toeplitz, [9], and later $\mathrm{Köthe}$, in a series of papers of which [10] is representative, studied these spaces for the case where $E$ is the space of natural numbers with the discrete topology and $\mu(n)=1$ for every natural number $n$. Dieudonné, [4], extended the theory to the case for which $E$ is $\sigma$-compact. Köthe spaces, which are also Banach spaces, were studied by Lorentz and Wertheim [7] for the case where $E=[0,1]$ and $\mu$ is Lebesgue measure. Examples of Köthe spaces are the Lebesgue spaces $L_{p}$, the Orlicz spaces $L_{\Phi}$, and arbitrary intersections of such spaces.

For each Köthe space $\Lambda$, the associated space $\Lambda^{*}$ determines a family of topologies in $\Lambda$. These topologies are locally convex, Hausdorff, and are compatible with the order relation in $\Lambda$. Among them there is a weakest and a strongest. It should be observed that the strongest of these topologies may be strictly stronger than the Mackey topology $m\left(\Lambda, \Lambda^{*}\right),[2]$. In the case that $E$ is $\sigma$-compact Dieudonné [4] showed that the Köthe space $\Lambda$ is complete for each of these topologies. Later, Goffman [5], using the work of Nakano, observed that the restriction of $\sigma$-compactness is not necessary.

In this paper a characterization is obtained of those Köthe spaces which, with their strongest Köthe topologies $S\left(\Lambda, \Lambda^{*}\right)$, are Banach spaces. A slight modification of these conditions gives a characterization of those Köthe spaces $\Lambda$, which, with their strongest Köthe topologies $S\left(\Lambda, \Lambda^{*}\right)$, are Fréchet spaces. It is shown that $\Lambda$ with the

Presented to the Society, November 27, 1959; received by the editors September 12, 1959 and, in revised form, October 10, 1959.

1 This paper is a portion of a thesis, directed by Dr. C. Goffman, to be submitted to Purdue University in partial fulfillment of the requirements for the degree of Doctor of Philosophy. 
topology $S\left(\Lambda, \Lambda^{*}\right)$ is a Banach space if and only if $\Lambda^{*}$ is a Banach space with the topology $S\left(\Lambda^{*}, \Lambda\right)$. If $\Lambda$ with the topology $S\left(\Lambda, \Lambda^{*}\right)$ is a Fréchet space, but not a Banach space, then $\Lambda^{*}$ is not metrizable for the topology $S\left(\Lambda^{*}, \Lambda\right)$. As $\Lambda^{*}$ is not in general the topological dual of $\Lambda$ with the topology $S\left(\Lambda, \Lambda^{*}\right)$ these results do not follow from the standard theory. In general the Köthe space $\Lambda$ has more than one Köthe topology. In $\S 5$ is considered a case where this is not so.

2. Let $W$ be a family of weakly bounded subsets of $\Lambda^{*}$ whose union is all of $\Lambda^{*}$. The weak duality determined for $\Lambda$ and $\Lambda^{*}$ by the bilinear form

$$
(f, g)=\int f g d \mu,
$$

$f \in \Lambda, g \in \Lambda^{*}$ makes it possible to define for $\Lambda$ the topology of uniform convergence on sets in $W$. With this topology $\Lambda$ is a locally convex space. If the sets in $W$ are normal ${ }^{2}$ as well as weakly bounded then this topology is called a Köthe topology and is denoted by $\mathscr{K}_{w}\left(\Lambda, \Lambda^{*}\right)$. The strongest and weakest such topologies are determined when $W$ contains all weakly bounded normal subsets of $\Lambda^{*}$ and $W$ contains only the normal closures ${ }^{3}$ of points in $\Lambda^{*}$ respectively. The weak topology $\sigma\left(\Lambda, \Lambda^{*}\right)$ is in general weaker than the weakest Köthe topology.

3. For a nonempty class $C$ of positive integrable functions $c(t)$ on $[0,1]$, Lorentz and Wertheim [7], define the Köthe space $X(C)$ as the set of measurable functions $f(t)$ for which

$$
\|f\|=\sup _{c \in C} \int_{0}^{1}|f(t)| c(t) d t<\infty .
$$

They show that if certain additional assumptions are placed on the set $C$, then their definition is equivalent to that given by Dieudonné, and their space $X(C)$ is a Banach lattice. Their additional conditions give the motivation for the following definition.

A nonempty subset $A$ of the Köthe space $\Lambda$ is said to satisfy condition $\left({ }^{*}\right)$, if for each nondecreasing sequence $\left(f_{n}\right)$ of nonnegative functions in $A$, there exists a function $f$ in $A$ such that $f \geqq f_{n}$ for $n=1,2, \cdots$.

${ }^{2}$ A subset $A$ of partially ordered vector space $X$ is normal if $x \in X, y \in A$, and $|x| \leqq|y|$ implies $x \in A$.

${ }^{3}$ The $P$ closure, of a subset $A$ of $\Lambda$, is the smallest subset of $\Lambda$ containing $A$ which is $P$. 
Normal convex subsets $A$ of $\Lambda$ which satisfy condition $\left(^{*}\right)$ will occur so of ten in the discussion that they will be referred to as admissible sets. Admissible sets play a fundamental role for:

Theorem 1. Every admissible subset of the Köthe space $\Lambda$ is weakly bounded.

Proof. Suppose that $A$ is an admissible subset of $\Lambda$ which is not weakly bounded. There exists a function $g$ in $\Lambda^{*}$, and a sequence $\left(f_{n}\right)$ in $A$ such that

$$
\left|\int f_{n} g d \mu\right|>2^{n}, \quad n=1,2, \cdots \text {. }
$$

Let $h_{n}=\sum_{i=1}^{n} 2^{-i}\left|f_{i}\right|$. Because $h_{n+1} \geqq h_{n} \geqq 0$ for each integer $n$, and because $A$ is admissible there exists a function $h$ in $A$ such that $h \geqq h_{n}$ for $n=1,2, \cdots$. Now

$$
\int|h g| d \mu \geqq \int\left|h_{n} g\right| d \mu>n, \quad n=1,2, \cdots .
$$

This implies that $|h g|$ is not integrable, while $h g$ is integrable. Hence $A$ is weakly bounded.

For a subset $A$ of the Köthe space $\Lambda$,

$$
A^{0}=\left\{g \in \Lambda^{*}:\left|\int f g d \mu\right| \leqq 1 \text { for all } f \in A\right\} .
$$

If $A$ is an absorbing subset of $\Lambda$, then $A^{0}$ is a weakly bounded subset of $\Lambda^{*}$. If $A$ is a weakly bounded subset of $\Lambda$, then $A^{0}$ is an absorbing subset of $\Lambda^{*}$. For proofs of these facts together with the fact that $\left(A^{0}\right)^{0}=A^{00}$ is the weak convex closure of $A$, see [2]

A consequence of the following lemma is that every Köthe topology $\varkappa_{w}\left(\Lambda, \Lambda^{*}\right)$ is compatible [14] with the natural order relation in $\Lambda$.

Lemma 1. If $A$ is a normal subset of the Köthe space $\Lambda$, then $A^{0}$ is a normal subset of $\Lambda^{*}$.

Proof. Observe first that

$$
\sup _{f \in A}\left|\int f g d \mu\right|=\sup _{f \in A} \int|f g| d \mu
$$

for any $g$ in $A^{0}$, that is, if $g$ is in $A^{0}$, then $|g|$ is in $A^{0}$. If $h$ is in $\Omega$ and $|h| \leqq|g|$ for some $g$ in $A^{0}$, then 


$$
1 \geqq \sup _{f \in A} \int|f g| d \mu \geqq \sup _{f \in A} \int|f h| d \mu .
$$

Hence $h$ is in $A^{0}$. It follows that $A^{0}$ is normal.

Theorem 2. Suppose that $A$ is a weakly bounded subset of the Köthe space $\Lambda$. If $\tilde{A}$ is the normal convex closure of $A$, then $(\tilde{A})^{00}$ satisfies condition $(*)$.

Proof. The set $(\tilde{A})^{00}$ is by [4] weakly bounded and by Lemma 1 normal. From previous remarks $(\widetilde{A})^{00}$ is also convex and weakly closed.

Suppose that $\left(f_{n}\right)$ is any nondecreasing sequence of nonnegative functions in $(\tilde{A})^{00}$. For any function $g$ in $\Lambda^{*}$ there exists a positive constant $M(g)$ such that

$$
\int f_{n}|g| d \mu \leqq M(g), \quad n=1,2, \cdots .
$$

Let $f=\lim f_{n}$ so that $f|g|=\lim f_{n}|g|$. By Fatou's lemma

$$
\int f|g| d \mu \leqq \liminf \int f_{n}|g| d \mu \leqq M(g) .
$$

Since the characteristic functions of compact subsets of $E$ are in the associated space $\Lambda^{*}$, the function $f$ is locally integrable, and hence is in $\Omega(E, \mu)$. As the function $g$ was chosen arbitrarily from $\Lambda^{*}$, (1) shows that $f$ is in $\Lambda$. It remains to show that $f$ is a weak limit of the sequence $\left(f_{n}\right)$, and hence is in $(\tilde{A})^{00}$.

To this end, suppose that a positive number $\epsilon$ and a function $g$ from $\Lambda^{*}$ have been chosen arbitrarily. Because $f|g|$ is integrable, there exists a compact subset $K$ of $E$, such that

$$
\int_{C(K)} f|g| d \mu \leqq \frac{\epsilon}{6} .
$$

A positive number $\delta$ exists such that for every measurable subset $F$ of $K$ for which $\mu(F)<\delta$,

$$
\int_{F} f|g| d \mu<\frac{\epsilon}{6} .
$$

By Egoroff's theorem, a measurable subset $F$ of $K$ exists, such that $\mu(F)<\delta$, and $f_{n}$ converges uniformly to $f$ on $K \sim F$. Choose a number $N$ such that $n \geqq N$ implies that $\left|f g-f_{n} g\right|<\epsilon / 3 \mu(K \sim F)$. It follows that 


$$
\left|\int\left(f-f_{n}\right) g d \mu\right| \leqq \int\left|f g-f_{n} g\right| d \mu<\epsilon, \quad n \geqq N .
$$

As $\epsilon$ and $g$ were chosen arbitrarily,

$$
\lim _{n \rightarrow \infty}\left|\int\left(f-f_{n}\right) g d \mu\right|=0
$$

for every $g$ in $\Lambda^{*}$. Hence $f$ is the weak limit of the sequence $\left(f_{n}\right)$.

Corollary 1. A normal subset $A$ of the Köthe space $\Lambda$ is weakly bounded if and only if $(\tilde{A})^{00}$ satisfies condition $\left(^{*}\right)$.

One obtains from this corollary that $\mathcal{K}_{w}\left(\Lambda, \Lambda^{*}\right)=S\left(\Lambda, \Lambda^{*}\right)$, if $W$ contains all admissible subsets of $\Lambda^{*}$.

4. It is known: if $X$ and $Y$ are two linear spaces in duality, then every weakly bounded subset of $X$ is bounded for the Mackey topology $m(X, Y),[2]$. For a Köthe space $\Lambda$ it was shown by Dieudonné in [4] that the bounded subsets for the weak topology $\sigma\left(\Lambda, \Lambda^{*}\right)$ are the same as the bounded subsets for the strong topology. His proof is based on results of Mackey $[12 ; 13]$. A simple direct proof will now be given.

LemMA 2. Every weakly bounded subset of the Köthe space $\Lambda$ is bounded for the strong topology $S\left(\Lambda, \Lambda^{*}\right)$.

Proof. Suppose the weakly bounded subset $A$ of $\Lambda$ is not bounded for the topology $S\left(\Lambda, \Lambda^{*}\right)$. By Corollary 1 an admissible subset $B$ of $\Lambda^{*}$ and a sequence $\left(f_{n}\right)$ in $A$ exist such that for each integer $n$ a function $g_{n}$ in $B$ can be found for which

$$
\int\left|g_{n} f_{n}\right| d \mu \geqq n 2^{n}
$$

Set $h_{n}=\sum_{k=1}^{n} 2^{-k}\left|g_{k}\right|$ for $n=1,2, \cdots$ As $\left(h_{n}\right)$ is a nondecreasing sequence of nonnegative functions in $B$, there exists a function $h$ in $B$ such that $h \geqq h_{n}$ for $n=1,2, \cdots$. The normal closure $\bar{A}$ of $A$ is weakly bounded [4], but

$$
\sup _{f \in \bar{A}}\left|\int f h d \mu\right| \geqq \int\left|f_{n} h\right| d \mu \geqq n
$$

for $n=1,2, \cdots$ implies that $\bar{A}$ is not weakly bounded. Hence the assumption that $A$ is not bounded for the topology $S\left(\Lambda, \Lambda^{*}\right)$ is false. 
Lemma 3. If $A$ is an absorbing admissible subset of the Köthe space $\Lambda$, then $A^{00}$ is a bounded neighborhood of zero for the strong topology.

Proof. Because $A$ is normal and absorbing $A^{0}$ is normal and weakly bounded. It follows that $A^{00}$ is a neighborhood of zero for the topology $S\left(\Lambda, \Lambda^{*}\right)$.

Since $A$ is admissible, it is by Theorem 1 weakly bounded. Therefore, $A^{0}$ is absorbing. But $A^{0}$ being absorbing implies that $A^{00}$ is weakly bounded. It follows from Lemma 2 that $A^{00}$ is bounded for the strong topology.

THEOREM 3. The Köthe space $\Lambda$ is a Banach space for the strong topology if and only if $\Lambda$ contains an absorbing admissible set.

Proof. Suppose that $A$ is an absorbing admissible subset of $\Lambda$, then $A^{00}$ is a convex bounded neighborhood of zero for the strong topology $S\left(\Lambda, \Lambda^{*}\right)$. By a theorem of Kolmogoroff [9] this topology is equivalent to a norm topology. Since $\Lambda$ is complete for $S\left(\Lambda, \Lambda^{*}\right)$ [5], it is a Banach space.

Conversely if $\Lambda$ is a Banach space for $S\left(\Lambda, \Lambda^{*}\right)$ then the unit sphere $A$ in $\Lambda$ is a weakly bounded absorbing set. It follows from Theorem 2 that $(A)^{00}$ is an absorbing admissible subset of $\Lambda$.

Corollary 2. The Köthe space $\Lambda$ is a Banach space for the topology $S\left(\Lambda, \Lambda^{*}\right)$ if and only if $\Lambda^{*}$ is a Banach space for the topology $S\left(\Lambda^{*}, \Lambda\right)$.

Proof. Suppose that $\Lambda$ is a Banach space for the topology $S\left(\Lambda, \Lambda^{*}\right)$ and let $A$ be an absorbing admissible subset of $\Lambda$. By Lemma $1, A^{0}$ is normal. As $A$ is weakly bounded and absorbing, $A^{0}$ is absorbing and weakly bounded. It is clear that $A^{0}$ is convex; therefore, $A^{0}$ is an admissible absorbing subset of $\Lambda^{*}$. Hence $\Lambda^{*}$ is a Banach space.

The converse is proved by interchanging the roles of $\Lambda$ and $\Lambda^{*}$ in the preceding argument.

Corollary 2 shows that the statement of Theorem 3 could be made in terms of the subsets of $\Lambda^{*}$. With this alternate approach in mind one states:

TheOREM 4. The Köthe space $\Lambda$ is a Fréchet space for the strong topology if and only if $\Lambda^{*}$ contains a sequence $B_{1} \subset B_{2} \subset \ldots$ of admissible subsets, such that every weakly bounded subset $B$ of $\Lambda^{*}$ is contained in one of the $B_{k}$.

Proof. Suppose $\Lambda^{*}$ contains such a sequence $B_{1} \subset B_{2} \subset \cdots$ of admissible sets. The topology of uniform convergence on these sets is clearly the strong topology for $\Lambda$. As this topology has a countable 
base for the neighborhood system of zero, it is a metric topology. The completeness of $\Lambda$ with this topology follows from [5].

Conversely, suppose that $\Lambda$ is a Fréchet space for the strong topology. A sequence $A_{1} \supset A_{2} \supset \ldots$ of normal convex subsets of $\Lambda$ can be found, which is a base in this topology for the neighborhood system of zero. The sets in the sequence $A_{1}^{0} \subset A_{2}^{0} \subset \cdots$ are admissible, for, each $A_{i}$ is both normal and absorbing. This sequence satisfies the further condition that each weakly bounded subset $B$ of $\Lambda^{*}$ is contained in one of the $A_{\mathbf{k}}^{0}$. For, if $B$ is a weakly bounded subset of $\Lambda^{*}$, and $\widetilde{B}$ is its normal closure, then $\widetilde{B}^{0}$ is a neighborhood of zero in $\Lambda$. This implies an integer $k$ can be found such that $A_{k} \subset \widetilde{B}^{0}$. Hence $B \subset A_{\mathrm{k}}^{0}$.

REMARK. If $\Lambda$ is a Fréchet space with the strong topology, Corollary 2 suggests asking, is $\Lambda^{*}$ a Fréchet space with its strong topology. The following discussion shows that if $\Lambda$ is a Fréchet space, but not a Banach space, then $S\left(\Lambda^{*}, \Lambda\right)$ is not a metric topology. Suppose $\Lambda^{*}$ with $S\left(\Lambda^{*}, \Lambda\right)$ is a Fréchet space even though $\Lambda$ with $S\left(\Lambda, \Lambda^{*}\right)$ is not a Banach space. By Theorem 4 a sequence $B_{1} \subset B_{2} \subset \cdots$ of admissible subsets of $\Lambda^{*}$ can be found whose union is $\Lambda^{*}$. The sets $B_{1}^{00}, B_{2}^{00}, \ldots$ are again admissible, and in addition are strongly closed. As the union of the sets $B_{i}^{00}$ is again $\Lambda^{*}$, the Baire category theorem ensures the existence of an integer $k$ such that $B_{\boldsymbol{k}}^{00}$ has an interior point. Thus $B_{\boldsymbol{k}}^{00}$ is an absorbing admissible set, that is, $\Lambda^{*}$ is a Banach space. By Corollary 2 this implies that $\Lambda$ is a Banach space, contrary to assumption. Thus, $S\left(\Lambda^{*}, \Lambda\right)$ is not a metric topology.

5. A strong unit $u$ in a partially ordered vector space $X$ is an element such that for each $x$ in $X$, there exists a constant $\lambda$ for which $x \leqq \lambda u$.

TheOREM 5. If the Köthe space $\Lambda^{*}$ contains a strong unit, then $\Lambda$ has a unique Köthe topology, and with this topology $\Lambda$ is a Banach space.

Proof. Let $u \geqq 0$ be a strong unit in $\Lambda^{*}$. Set $B=\left\{g \in \Lambda^{*}:|g| \leqq u\right\}$. If $g$ is in $\Lambda^{*}$, then a constant $\lambda$ exists such that $g$ is in $\lambda B$, that is, $B$ is an absorbing subset of $\Lambda^{*}$. Clearly the set $B$ is admissible. Hence $\Lambda$ is a Banach space for the topology $S\left(\Lambda, \Lambda^{*}\right)$.

Let $\mathcal{K}_{w}\left(\Lambda, \Lambda^{*}\right)$ be any Köthe topology for $\Lambda$. There exists a normal set $V$ in $W$ such that $u$ is in $V$. Thus $B \subset V$, from which it follows that $\mathfrak{K}_{w}\left(\Lambda, \Lambda^{*}\right)$ is finer than $S\left(\Lambda, \Lambda^{*}\right)$. As $S\left(\Lambda, \Lambda^{*}\right)$ is always finer than $\mathcal{K}_{w}\left(\Lambda, \Lambda^{*}\right)$, these two topologies must be the same.

The existence of a unique Köthe topology on $\Lambda$ does not imply 
that $\Lambda^{*}$ has a strong unit. For consider the Köthe space $\Omega$ of all real sequences. Its associate, $\Phi$, is the space of sequences which are zero except at a finite number of places. This space has no strong unit. However, $\Omega$ has only one Köthe topology. Note that the weak topology for $\Omega$, that is the topology of pointwise convergence, is also the weakest Köthe topology. This topology is compatible with the order relation in $\Omega$ and is also a metric topology. Hence by [6] or [8] it is the finest compatible topology. Since $S(\Omega, \Phi)$ is a compatible topology it is weaker than the weak topology $\sigma(\Omega, \Phi)$. Thus these two topologies are the same, that is, $\Omega$ has only one Köthe topology.

\section{REFERENCES}

1. G. Birkhoff, Lattice theory, Amer. Math. Soc. Colloquium Publications vol. 25, 1948.

2. N. Bourbaki, Espaces vectoriels topologiques, Actualités Sci. Ind. No. 1229, 1955.

3. - Integration, Actualités Sci. Ind. vol. 1175 (1952). 115.

4. J. Dieudonné, Sur les espaces de Köthe, J. Analyse Math. No. 1, 1951, pp. 81-

5. C. Goffman, Completeness in topological vector lattices, Amer. Math. Monthly vol. 66 (1959) pp. 87-92.

6. - Compatible semi-norms in a vector lattice, Proc. Nat. Acad. Sci. U.S.A. vol. 42 (1956) pp. 782-783.

7. G. Lorentz and D. Wertheim, Representation of linear functionals on Köthe spaces, Canad. J. Math. vol. 5 (1953) pp. 568-575.

8. J. Kist, Locally 0-convex spaces, Duke Math. J. vol. 25 (1958) pp. 569-582.

9. A. Kolmogoroff, Zur Normierbarkeit eines allgemeinen topologischen linearen Raumes, Studia Math. vol. 5 (1934) pp. 29-33.

10. G. Köthe and O. Toeplitz, Lineare Räume mit unendlich vielen koordinaten, J. Reine Angew. Math. vol. 171 (1934) pp. 193-226.

11. G. Köthe, Neubegründung der theorie der vollkommen Räume, Math. Nachr. vol. 4 (1951) pp. 70-80.

12. G. W. Mackey, On infinite dimensional linear spaces, Trans. Amer. Math. Soc. vol. 57 (1945) pp. 155-207.

13. - On convex topological linear spaces, Trans. Amer. Math. Soc. vol. 60 (1946) pp. 520-537.

14. I. Namioka, Partially ordered linear topological spaces, Memoirs Amer. Math. Soc. vol. 24, 1957.

Purdue University 